\title{
The immunomodulatory potential of in vitro digested low-fat milk supplemented with brewers' spent grain protein hydrolysate; selection of a non-cytotoxic level of digestate
}

\author{
D. J. Crowley ${ }^{1}$, Y. C. O’Callaghan ${ }^{1}$, A.L. McCarthy ${ }^{2}$, C. O. Piggott ${ }^{2}$, R. J. FitzGerald ${ }^{2}$ \\ and N. M. O'Brien ${ }^{1}$ \\ ${ }^{1}$ School of Food and Nutritional Sciences, University College Cork, Ireland and ${ }^{2}$ Department of Life Sciences, \\ University of Limerick, Ireland
}

Protein hydrolysates from agricultural crops have shown encouraging bioactive and techno-functional characteristics that may be used in the development of functional foods ${ }^{(1)}$. It is important that bioactive protein hydrolysates demonstrate an ability to retain their bioactivity during digestion. Brewers' spent grain (BSG), a by-product of the brewing industry, is a potential source for the development of protein hydrolysates. The aim of this study was to incorporate a bioactive, BSG-derived protein hydrolysate into commercially available low-fat milk and assess the cytotoxicity and immunomodulatory effects of digestates, following in vitro gastrointestinal digestion.

Hydrolysate $\mathrm{U}$ was obtained on Alcalase 2.4L digestion of a BSG protein-rich isolate. The hydrolysate was freeze-dried whole (U) or fractionated using 5 and $3 \mathrm{kDa}$ molecular cut-off membranes, and permeates and retentate were designated $\mathrm{U}, \mathrm{U}<3, \mathrm{U}<5$ and $\mathrm{U}>5$. Samples were added to low-fat milk at a concentration of $0 \cdot 125 \%(\mathrm{v} / \mathrm{v})$. A static gastrointestinal digestion model, as previously described $^{(2)}$ was used to mimic human digestion. The (3-(4,5-dimethylthiazol-2-yl)-2,5-diphenyltetrazolium bromide) (MTT) assay was used to assess the effect of digestates $(0-10 \% ; \mathrm{v} / \mathrm{v})$ on Jurkat $\mathrm{T}$ cell proliferation. The effect of digestates on interferon-gamma (IFN-gamma) and interleukin-6 (IL-6) secretion in concanavalin-A (con-A) stimulated Jurkat T cells was measured by ELISA. Data were expressed as a percentage of untreated (control) cells.

\begin{tabular}{|c|c|c|c|c|c|c|c|c|c|c|c|c|}
\hline & Contr & & \multicolumn{2}{|c|}{$1 \%$ digestate } & \multicolumn{2}{|c|}{$2 \%$ digestate } & \multicolumn{2}{|c|}{$2.5 \%$ digestate } & \multicolumn{2}{|c|}{$5 \%$ digestate } & \multicolumn{2}{|c|}{$10 \%$ digestate } \\
\hline & \multicolumn{12}{|c|}{ Cell Viability (\%) } \\
\hline & $\overline{\text { Mean }}$ & se & Mean & se & Mean & se & Mean & se & Mean & se & Mean & se \\
\hline Digestate blank & $95 \cdot 3$ & $5 \cdot 8$ & $108 \cdot 2$ & $2 \cdot 8$ & $121 \cdot 3$ & $7 \cdot 2$ & $120 \cdot 6$ & $14 \cdot 8$ & $117 \cdot 9$ & $6 \cdot 2$ & $126 \cdot 7$ & $3 \cdot 5$ \\
\hline Unfortified milk & $96 \cdot 9$ & $2 \cdot 8$ & $104 \cdot 1$ & $2 \cdot 6$ & $106 \cdot 6$ & $3 \cdot 2$ & $98 \cdot 3$ & $12 \cdot 8$ & $77 \cdot 0$ & $18 \cdot 5$ & $43 \cdot 6$ & $35 \cdot 2$ \\
\hline $\mathrm{U}$ & $92 \cdot 7$ & $7 \cdot 4$ & $102 \cdot 1$ & $7 \cdot 0$ & $110 \cdot 8$ & $4 \cdot 0$ & $104 \cdot 2$ & $3 \cdot 7$ & $77 \cdot 8$ & $19 \cdot 0$ & $23 \cdot 6^{*}$ & $7 \cdot 7$ \\
\hline $\mathrm{U}<3$ & $101 \cdot 4$ & 1.7 & $109 \cdot 3$ & $9 \cdot 2$ & $110 \cdot 2$ & $18 \cdot 0$ & $98 \cdot 1$ & 1.8 & $81 \cdot 1$ & $14 \cdot 7$ & $29 \cdot 1 *$ & $21 \cdot 0$ \\
\hline $\mathrm{U}<5$ & $112 \cdot 8$ & $6 \cdot 2$ & $100 \cdot 8$ & 4.9 & $103 \cdot 0$ & $7 \cdot 0$ & 98.9 & $10 \cdot 7$ & $73 \cdot 8$ & $17 \cdot 5$ & $33 \cdot 0 *$ & $27 \cdot 2$ \\
\hline $\mathrm{U}>5$ & 101.0 & 1.8 & 99.6 & $6 \cdot 6$ & $99 \cdot 4$ & $5 \cdot 0$ & $94 \cdot 0$ & 3.5 & $29 \cdot 7^{*}$ & $13 \cdot 8$ & $4 \cdot 2 *$ & 1.4 \\
\hline
\end{tabular}

Values are mean of three independent experiments. Statistical analysis by ANOVA followed by Dunnett's test

* Denote significant difference in cell viability, relative to untreated Jurkat cells $(P<0 \cdot 05)$.

Treatment with digestates of milk with added hydrolysates $\mathrm{U}, \mathrm{U}<3$ and $\mathrm{U}<5$ at $10 \%(\mathrm{v} / \mathrm{v})$ and hydrolysates $\mathrm{U}>5$ at $5 \%$ and $10 \%$ $(\mathrm{v} / \mathrm{v})$, for 24 hours significantly reduced viability of Jurkat $\mathrm{T}$ cells. Following on from the cytotoxicity results, the highest non-toxic concentration of digestates $(2 \cdot 5 \%)$ was selected for further investigation. Preliminary results suggest that milk digestates with added $>5 \mathrm{kDa}$ hydrolysate can decrease IFN-gamma and IL-6 secretion in stimulated Jurkat $\mathrm{T}$ cells (data not shown). In conclusion, these results suggest that low-fat milk fortified with BSG hydrolysate can attenuate cytokine production in stimulated Jurkat T cells.

This research was supported through the Food Institutional Research Measure, administered by the Department of Agriculture, Food and the Marine, Ireland.

1. McCarthy AL, O'Callaghan YC, \& O'Brien NM. (2013) Agriculture 3, 112-130.

2. McCarthy AL, O'Callaghan YC, Neugart S et al. (2013) Food Chem 141, 2567-2574. 